\title{
Applicability of the orientation average formula in heavy-ion fusion reactions of deformed nuclei
}

\author{
Tamanna Rumin ${ }^{1},{ }^{*}$ Kouichi Hagino ${ }^{2},{ }^{\dagger}$ and Noboru Takigawa ${ }^{1} \ddagger$ \\ ${ }^{1}$ Department of Physics, Tohoku University, Sendai 980-8578, Japan \\ ${ }^{2}$ Institute for Nuclear Theory, Department of Physics, University of Washington, Seattle, Washington 98195
}

(October 29, 2018)

\begin{abstract}
In heavy-ion fusion reactions involving a well deformed nucleus, one often assumes that the orientation of the target nucleus does not change during the reaction. We discuss the accuracy of this procedure by analyzing the excitation function of the fusion cross section and the fusion barrier distribution in the reactions of ${ }^{154} \mathrm{Sm}$ target with various projectiles ranging from ${ }^{12} \mathrm{C}$ to ${ }^{40} \mathrm{Ar}$. It is shown that the approximation gradually looses its accuracy with increasing charge product of the projectile and target nuclei because of the effects of finite excitation energy of the target nucleus. The relevance of such inaccuracy in analyzing the experimental data is also discussed.
\end{abstract}

25.60.Pj, 21.60.Ev, 24.10.Eq, 25.

It is now well established that nuclear intrinsic degrees of freedom strongly influence the fusion cross section in heavy-ion reactions at energies near and below the Coulomb barrier [1,2]. Typical examples include the rotational excitation of a deformed target nucleus which leads to a large enhancement of the fusion cross section at low energies. A characteristic in this case is that the excitation energy of the rotational motion is often much smaller than the curvature of the fusion barrier, which determines the time scale of the fusion process. This is the case when, e.g., one of the deformed rare earth nuclei or actinides is the target nucleus. One then often calculates the fusion probability for each partial wave $J$ following [3, 1 ]

$$
P_{J}^{S}(E)=\int_{0}^{1} P_{J}(E, \theta) d(\cos \theta)
$$

where $\theta$ is the orientation of the deformed target, which will be specified later more precisely. We call this the orientation average formula. The upper index $S$ stands for the sudden tunneling approximation. $P_{J}(E, \theta)$ is the fusion probability for a given orientation. It is determined by solving a one-dimensional Schrödinger equation for the relative distance between the projectile and target $R$, where the potential consists of the nuclear and Coulomb components given by

$$
\begin{aligned}
V_{N}(R, \theta) & =\frac{-V_{0}}{1+\exp \left[\left(R-R_{P}-R(\theta)\right) / a\right]} \\
V_{C}(R, \theta) & =\frac{Z_{P} Z_{T} e^{2}}{R}+\sum_{\lambda}\left[\beta_{\lambda}+\frac{2}{7} \sqrt{\frac{5}{\pi}} \beta_{\lambda}^{2} \delta_{\lambda, 2}\right] \frac{3 Z_{P} Z_{T} e^{2}}{2 \lambda+1} \frac{R_{T}^{\lambda}}{R^{\lambda+1}} Y_{\lambda 0}(\theta)
\end{aligned}
$$

with the angle dependent radius of the target nucleus

$$
R(\theta)=R_{T}\left[1+\sum_{\lambda} \beta_{\lambda} Y_{\lambda 0}(\theta)\right]
$$

In these equations $V_{0}$ is the depth parameter of the nuclear potential between the projectile and target, $R_{P}\left(R_{T}\right)$ and $Z_{P}\left(Z_{T}\right)$ are the radius and the atomic number of the projectile (target), $\beta_{\lambda}$ is the deformation parameter of the target nucleus of multipolarity $\lambda$, and $a$ the surface diffuseness parameter. The second order term is retained only for the quadrupole coupling in the Coulomb interaction. As in almost all analyses of heavy-ion fusion reactions at sub-barrier energies, we adopt the no-Coriolis approximation and work in the rotating coordinate frame [5] 7], where the $\mathrm{z}$-axis is taken to be parallel to the radial vector of the relative motion between the projectile and the target

*Electronic address: rumin@nucl.phys.tohoku.ac.jp

${ }^{\dagger}$ Electronic address: hagino@phys.washington.edu

${ }^{\ddagger}$ Electronic address: takigawa@nucl.phys.tohoku.ac.jp 
nuclei. Also, we assume an axial symmetry for the target nucleus. The parameter $\theta$ is then the angle between the symmetry axis and the z-axis in the rotating frame.

Eq. (11) is quite appealing and provides a simple understanding of the large enhancement of the fusion cross section at sub-barrier energies. It states that the deformation and the associated rotational excitation of the target nucleus result in a distributed fusion barriers, where some of them will be lower than that of the bare potential in the absence of these degrees of freedom. The same idea of the orientation average is still correct if one replaces the Gauss integral in Eq. (1) by an appropriate summation even when the rotational band is truncated at a certain angular momentum $I_{\max }$ as is always the case in nuclear physics. If only $\lambda=2$ is present, the corresponding sum is given by the $I_{\text {max }}+2$ points Gauss quadrature [3].

The orientation average procedure is exact only in the degenerate spectrum limit, i.e., when the excitation energy of the rotational motion is zero, which is not the case in actual nuclei. It is, therefore, important to examine the applicability of this formula before one makes a quantitative analysis of the experimental data. A step towards this direction has been undertaken in Ref. [4], where an analytic formula has been derived to modify the fusion probability in the sudden tunneling approximation by taking the finite excitation energy of the rotational motion into account as

$$
P_{J}(E) \approx \exp \left[-\frac{4}{15}\left(\frac{\sqrt{\frac{5}{4 \pi}} \beta_{2} F\left(R_{B}\right) T_{0}}{\hbar}\right)^{2} \frac{\epsilon_{2^{+}}^{*} T_{0}}{\hbar}\right] P_{J}^{S}(E)
$$

where $\epsilon_{2+}^{*}$ is the excitation energy of the first excited $2^{+}$state of the rotational band. $T_{0}=\pi / \Omega, \Omega$ being the curvature of the fusion barrier, is the tunneling time and $F$ is a measure of the strength of the channel coupling which causes the rotational excitation of the target nucleus during the collision. In Ref. [4], it was identified with the coupling strength at the fusion barrier $R_{B}$.

Eq. (5) is useful to qualitatively discuss the conditions to apply the orientation average formula. It clearly shows that the excitation energy of the rotational motion should be much smaller than the curvature of the fusion barrier. It also shows that the coupling strength also governs the validity of the formula. Despite these advantages, as we see later, Eq. (5) is not tolerable for quantitative discussions. This is partly because Eq. (5) has been derived in a perturbation theory, and also by ignoring the radial dependence of the coupling form factor. In heavy-ion fusion reactions, the latter is a very crude approximation, because the nuclear and Coulomb couplings interfere leading to a strong radial dependence of the coupling form factor [8]. Nevertheless, for future reference, we show in Table I the exponential factor in Eq. (5), which we call the dissipation factor, for several systems to be examined later. Table II lists the bare nuclear potential parameters we used and the Coulomb barrier properties, where the radius parameter $r_{0}$ has been introduced as $R_{P}+R_{T}=r_{0}\left(A_{P}^{1 / 3}+A_{T}^{1 / 3}\right)$.

In this paper we compare the results of the orientation average formula with the numerical solutions of the corresponding coupled channels equations, which are obtained using the computer code CCFULL 9] by keeping the finite excitation energy, i.e. $0.082 \mathrm{MeV}$, of the target nucleus ${ }^{154} \mathrm{Sm}$. We call the latter as the exact coupled channels calculations. We keep only the quadrupole deformation in the target, i.e., $\lambda=2$, for simplicity. All the projectiles are treated as inert. We truncate the rotational band of ${ }^{154} \mathrm{Sm}$ at $I_{\max }=20^{+}$member for all reactions. According to the table of isotopes [10], the highest member which has been so far experimentally observed is $16^{+}$. We have confirmed that the comparison between the exact coupled-channels and the orientation average calculations does not almost change beyond $I_{\max }=12^{+}$. We also remark that the coupled-channels calculations almost converge at $I_{\max }=12^{+}$ member 111 for the ${ }^{16} \mathrm{O}$ and ${ }^{154} \mathrm{Sm}$ reactions, but higher levels introduce non-negligible effects for heavy projectiles.

Both the orientation average and the exact coupled channels calculations give the fusion cross section which is a monotonically increasing function of energy. In order to facilitate to see the accuracy of the orientation average formula, we plot in Fig. 1 the ratio of the fusion cross section calculated by solving the exact coupled channels equations to that obtained by the orientation average formula. The figure clearly shows that the deviation of the results of the orientation average formula from those of the exact calculations gradually increases with the charge product of the projectile and target nuclei. This behaviour is consistent with Table I, which has been obtained based on Eq. (5), though the actual deviation is much larger than what Eq. (5) predicts. We also note that the deviation is significant even for light projectiles at energies below the Coulomb barrier.

An important question is whether this significantly affects the understanding of the mechanism of heavy-ion fusion reactions and the structural informations such as the deformation parameters extracted from the data analyses. In this connection, we compare in Fig. 2 the experimental data of the excitation function of the fusion cross section and the fusion barrier distribution [12,13, with the theoretical results calculated by the orientation average formula (the dashed line) and by the exact treatment of the corresponding coupled-channels equations (the solid line) for ${ }^{16} \mathrm{O}+{ }^{154} \mathrm{Sm}$ reaction. The second derivative of the cross section times the bombarding energy has been calculated with the point difference method with the interval $\Delta E=2 \mathrm{MeV}$. Similar comparison is done in Fig. 3 for ${ }^{40} \mathrm{Ar}+{ }^{154} \mathrm{Sm}$ 
reaction. The bare Coulomb barrier is at $V_{B}=59.41 \mathrm{MeV}$ and $127.57 \mathrm{MeV}$ for ${ }^{16} \mathrm{O}+{ }^{154} \mathrm{Sm}$ and ${ }^{40} \mathrm{Ar}+{ }^{154} \mathrm{Sm}$ reactions, respectively. In these calculations the deformation parameter $\beta_{2}$ of ${ }^{154} \mathrm{Sm}$ was determined to be 0.32 by fitting the data of ${ }^{16} \mathrm{O}+{ }^{154} \mathrm{Sm}$ fusion reactions. The same deformation parameter has been used for ${ }^{40} \mathrm{Ar}+{ }^{154} \mathrm{Sm}$ reactions as well, though the effective optimum values can differ in two reactions. Fig. 2 shows that the difference between two theoretical lines is much smaller than their deviation from the experimental data. This indicates that one can safely use the orientation average formula to data analyses of ${ }^{16} \mathrm{O}+{ }^{154} \mathrm{Sm}$ fusion reactions, even though its deviation from the exact calculation can be noticeable at low energies as shown in Fig. 1. The top panel of Fig. 3 shows that the deviation between the exact and the orientation average calculations is not so drastic even for ${ }^{40} \mathrm{Ar}+{ }^{154} \mathrm{Sm}$ reaction in this semilogarithmic plot. On the other hand, the situation is different for the fusion barrier distribution shown in the lower panel. The difference between the two calculations can be more easily recognizable than the case for ${ }^{16} \mathrm{O}+{ }^{154} \mathrm{Sm}$ fusion reactions. The present theoretical calculations still underestimate the fusion cross sections compared with the experimental data for the ${ }^{40} \mathrm{Ar}+{ }^{154} \mathrm{Sm}$ reaction at low energies. This will be partly because we ignored the projectile excitations, whose excitation energy is as small as $1.46 \mathrm{MeV}$.

In summary, we have studied the accuracy of the orientation average formula for fusion cross sections between a spherical projectile and a well deformed target by comparing its results with those of the exact coupled channels calculations. We found that the results of the orientation average formula significantly differ from those of the exact numerical solution of the corresponding coupled channels equations for systems with a large charge product of the projectile and target nuclei. This suggests the necessity of the proper coupled-channels calculations beyond the orientation average formula for these systems in order to properly identify the role of various channel coupling effects and to extract reliable informations on nuclear structure, especially through the analysis of the fusion barrier distribution of high precision data. Unexpectedly, we observed a significant deviation of the orientation average formula from the exact calculations even for light projectiles in the energy region well below the Coulomb barrier. This deviation is, however, much smaller than the deviation of these two calculations from the experimental data. In this sense, the orientation average formula is safely applicable to light systems.

\section{ACKNOWLEDGMENTS}

We thank D.M. Brink for useful discussions. This research was supported by the Monbusho Scholarship and the International Scientific Research Program: Joint Research: contract number 09044051 from the Japanese Ministry of Education, Science and Culture, and by the U.S. Department of Energy under Grant no. DE-FG03-00-ER41132.

[1] A. B. Balantekin, N. Takigawa, Rev. Mod. Phys. 70, 77 (1998).

[2] M. Dasgupta, D.J. Hinde, N. Rowley, and A.M. Stefanini, Annu. Rev. Nucl. Part. Sci., 48, 401 (1998).

[3] M.A. Nagarajan, A.B.Balantekin, N. Takigawa, Phys. Rev. C 34, 894 (1986).

[4] K. Hagino, N. Takigawa, J. R. Bennett and D. M. Brink, Phys. Rev. C 51, 3190 (1995).

[5] N. Takigawa and K. Ikeda, in Proceedings of the Symposium on The Many Facets of Heavy Ion Fusion Reactions, Argonne National Laboratory Report No. ANL-PHY- 87-1, 1986, p. 613.

[6] N. Takigawa, Y. Alhassid, and A. B. Balantekin, Phys. Rev. C 45, 1850 (1992).

[7] K. Hagino, N. Takigawa, A. B. Balantekin, and J. R. Bennett, Phys. Rev. C 52, 286 (1995).

[8] K. Hagino, N. Takigawa, and A.B. Balantekin, Phys. Rev. C 56, 2104 (1997).

[9] K. Hagino, N. Rowley, and A.T. Kruppa, Comput. Phys. Comm 123, 143 (1999).

[10] R.B. Firestone et al., Table of Isotopes Vol.II (John Wiley and Sons, 1996, New York)

[11] Tamanna Rumin, K. Hagino, and N. Takigawa, Phys. Rev. C 61, 14605 (2000).

[12] N. Rowley, G.R. Satchler, and P.H. Stelson, Phys. Lett. B 254, 25 (1991).

[13] J.R. Leigh, M. Dasgupta, D.J. Hinde, J.C. Mein, C.R. Morton, R.C. Lemmon, J.P. Lestone, J.O. Newton, H. Timmers, J.X. Wei, and N. Rowley, Phys. Rev. C 52, 3151 (1995).

[14] W. Reisdorf, F.P. Hessberger, K.D. Hildenbrand, S. Hofmann, G. Münzenberg, K.H. Schmidt, J.H.R. Schneider, W.F.W. Schneider, K. Sümmerer, G. Wirth, J.V. Kratz and K. Schlitt, Nucl. Phys. A 438, 212 (1985). 
TABLE I : Dependence of the dissipation factor on the projectile in the fusion of ${ }^{154} \mathrm{Sm}$ target estimated based on Eq. (5).

\begin{tabular}{|l|l|l|l|l|l|l|l|l|}
\hline Reactions & ${ }^{12} \mathrm{C}$ & ${ }^{16} \mathrm{O}$ & ${ }^{20} \mathrm{Ne}$ & ${ }^{24} \mathrm{Mg}$ & ${ }^{28} \mathrm{Si}$ & ${ }^{32} \mathrm{~S}$ & ${ }^{36} \mathrm{Ar}$ & ${ }^{40} \mathrm{Ar}$ \\
\hline${ }^{154} \mathrm{Sm}$ & 0.810 & 0.689 & 0.567 & 0.456 & 0.357 & 0.274 & 0.207 & 0.164 \\
\hline
\end{tabular}

TABLE II : The bare nuclear potential parameters for different systems.

\begin{tabular}{|l|l|l|l|l|l|l|}
\hline Systems & $V_{0}(\mathrm{MeV})$ & $r_{0}(\mathrm{fm})$ & $a(\mathrm{fm})$ & $V_{B}(\mathrm{MeV})$ & $R_{B}(\mathrm{fm})$ & $\hbar \Omega(\mathrm{MeV})$ \\
\hline $12 \mathrm{C}+{ }^{154} \mathrm{Sm}$ & 150.0 & 0.950 & 1.05 & 44.60 & 10.81 & 3.47 \\
\hline${ }^{16} \mathrm{O}+{ }^{154} \mathrm{Sm}$ & 165.0 & 0.950 & 1.05 & 59.41 & 10.81 & 3.48 \\
\hline${ }^{20} \mathrm{Ne}+{ }^{154} \mathrm{Sm}$ & 190.0 & 0.945 & 1.05 & 73.85 & 10.87 & 3.48 \\
\hline${ }^{24} \mathrm{Mg}+{ }^{154} \mathrm{Sm}$ & 225.0 & 0.935 & 1.05 & 88.04 & 10.95 & 3.50 \\
\hline${ }^{28} \mathrm{Si}+{ }^{154} \mathrm{Sm}$ & 255.0 & 0.935 & 1.05 & 101.58 & 11.09 & 3.50 \\
\hline${ }^{32} \mathrm{~S}+{ }^{154} \mathrm{Sm}$ & 285.0 & 0.935 & 1.05 & 114.89 & 11.22 & 3.51 \\
\hline${ }^{36} \mathrm{Ar}+{ }^{154} \mathrm{Sm}$ & 315.0 & 0.935 & 1.05 & 128.00 & 11.34 & 3.51 \\
\hline${ }^{40} \mathrm{Ar}+{ }^{154} \mathrm{Sm}$ & 294.0 & 0.935 & 1.05 & 127.57 & 11.38 & 3.34 \\
\hline
\end{tabular}




\section{Figure Captions}

FIG. 1

The ratio of the fusion cross sections obtained in the exact coupled channels calculations to those obtained in the orientation average formula.

FIG. 2

Comparison of the experimental data and the results of the orientation average (the dashed line) and the exact coupled channels (the solid line) calculations for the ${ }^{16} \mathrm{O}+{ }^{154} \mathrm{Sm}$ fusion reaction. The top and bottom panels are the fusion excitation function and the fusion barrier distribution, respectively. The data are taken from Ref. [13].

FIG. 3

The same as Fig. 2, but for the ${ }^{40} \mathrm{Ar}+{ }^{154} \mathrm{Sm}$ fusion reaction. The data are taken from Ref. [14]. 


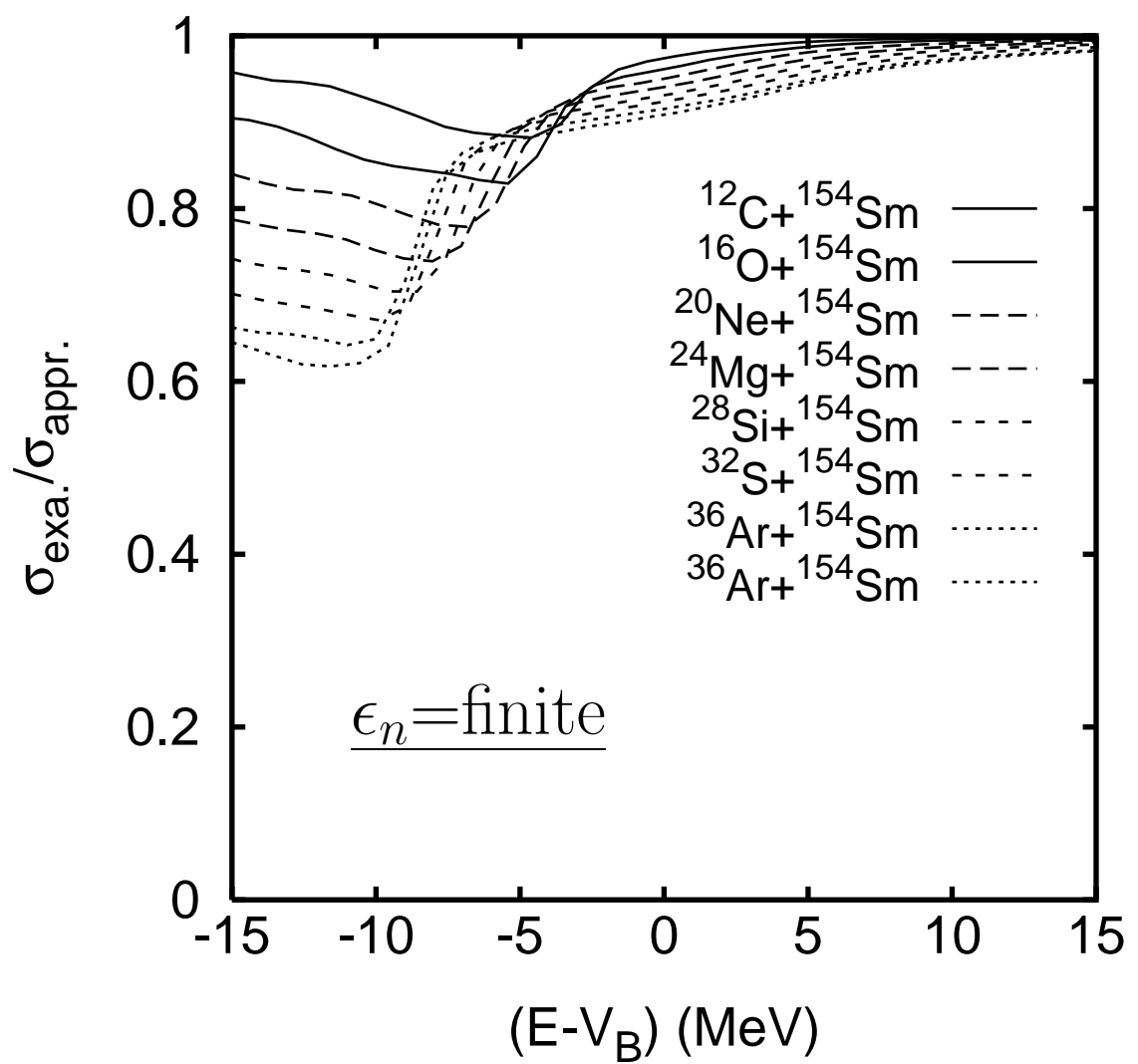

Fig. 1 

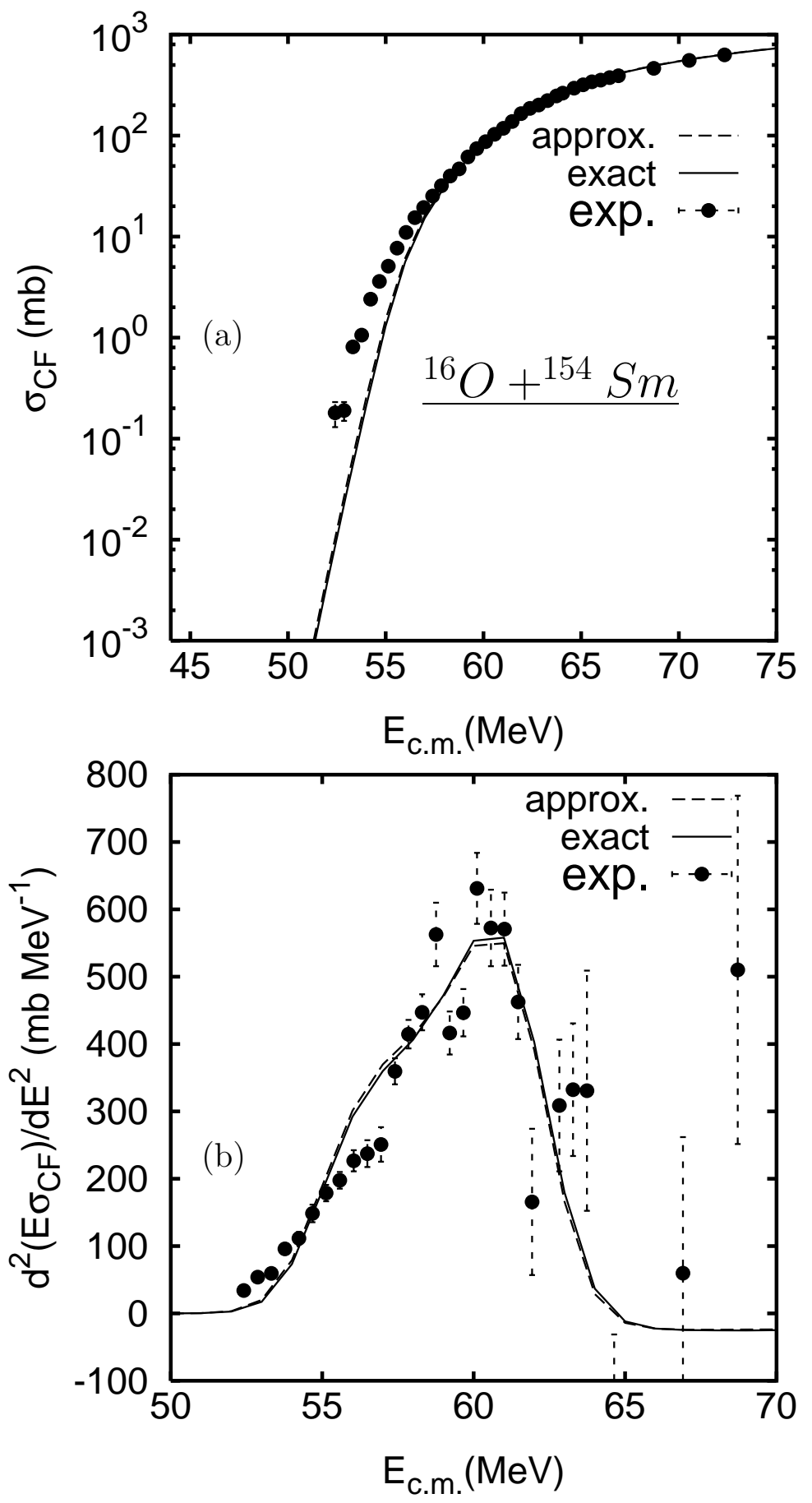

Fig. 2 

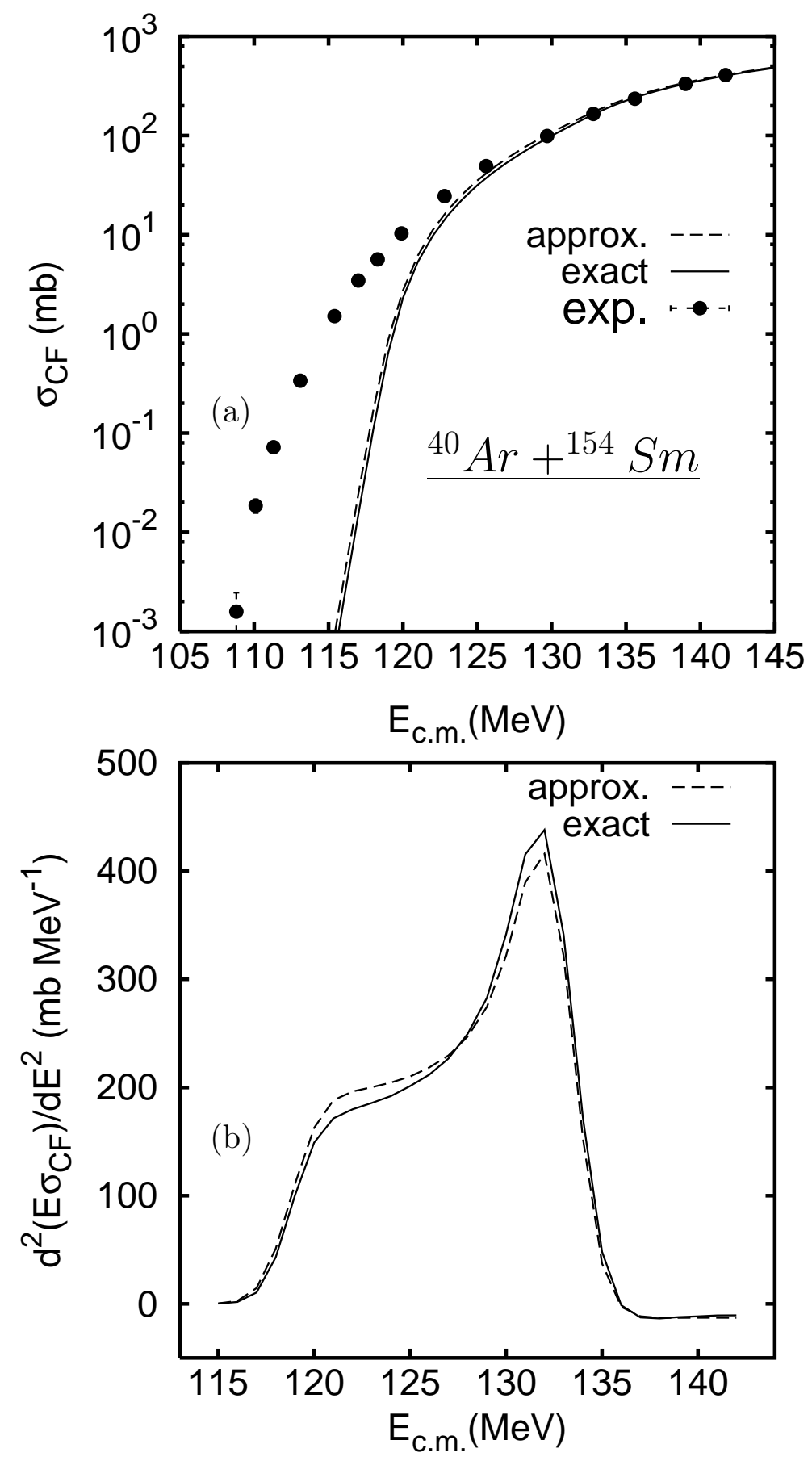

Fig. 3 